C.I.F. T. XI, Fasc. 1 y 2 (1985), págs. 91-122

\title{
NUEVAS VARIANTES DE LOS ROMANCES DE LUIS DE GONGORA
}

\author{
Ana Mercedes Alcolea Serrano* \\ Instituto Politécnico de F.P. de Teruel
}

\begin{abstract}
RESUMEN
Nuestro estudio trata de sacar a la luz las variantes de los Romances de D. Luis de Góngora y Argote que presenta en Manuscrito de la Biblioteca del Real Seminario de San Carlos de Zaragoza, con respecto a los manuscritos conocidos del poeta cordobés y a las ediciones manejadas por la crítica anterior.

El trabajo ofrece el repertorio de variantes de los Romances de Cóngora, abriendo así nuevos caminos para la investigación.
\end{abstract}

\begin{abstract}
Our article tries to show unknown variants about most of D. Luis de Góngora's Romances. The new versions belong to the texts of a manuscript titled Obras de Góngora, which is kept at the Real Seminario de San Carlos de Zaragoza. Some prestigious editions of Cordovan poet have been consulted for the collation of verses.

As a result of this research, we have found that there are important variants in the studied manuscript concerning known and handled versions used by previous critics.

The pattern of variants opens new possibilities of investigation about manuscript families in the Gongora's poems.
\end{abstract}

* Licenciada en Filología Hispánica. Residencia Universitaria, módulo 314; Ciudad Escolar; Teruel. Recibido el 10-7-1986. 


\section{INTRODUCCION}

Para el conocimiento exhaustivo de la obra de D. Luis de Góngora y Argote, es necesario recorrer todas y cada una de las versiones de sus obras que en diferentes manuscritos, cancioneros y ediciones circulaban en su época y posteriormente.

Con este trabajo pretendemos realizar una aportación al estudio de los Romances de D. Luis de Góngora, sacando a la luz las variantes que aparecen en un Manuscrito que se halla con la signatura B - 3 - 9 en la Biblioteca del Real Seminario de San Carlos de Zaragoza.

Para efectuar el cotejo nos hemos servido de las ediciones de los Romances gongorinos basadas en el Manuscrito Chacón, considerado como el más próximo a la versión definitiva del autor.

El Manuscrito de la Biblioteca del Real Seminario de San Carlos de Zaragoza presenta como título Obras de Góngora y no tiene datación, la única fecha que consta es la de 1619 en el epígrafe de un romance que conmemora la vuelta de Portugal del Rey Felipe III.

En el Manuscrito de San Carlos no aparecen todos los Romances que el Manuscrito Chacón atribuye a D. Luis de Góngora y sí tres cuya filiación se documenta como dudosa ("En buen hora, oh gran Phelipe", "Tú, noche que alivias" y "Busqué para enamorarme").

\section{Descripción del manuscrito}

Las características con que aparece descrito en el catálogo de manuscritos e incunables de la Biblioteca del Real Seminario de San Carlos de Zaragoza son las siguientes:

Manuscrito del siglo XVII, en papel a una columna, 303 hojas numeradas del 1 al 200 (23 en b.). Al margen llevan la dedicatoria o alguna explicación, aunque no todos. $280 \mathrm{~mm}$ x $195 \mathrm{~mm}$. Caja de escritura: 215 x $80 \mathrm{~mm}$. Encuadernación en pergamino. Tejuelo: Obras de Góngora. Número 9. 496.

Además de estas características podemos anotar las siguientes:

- Cubierta: piel

- Hojas de guarda: 7 delante y 11 detrás

- Hojas foliadas: 200 seguidas, numerada asimismo la ${ }^{\circ} 271$ con lápiz y grafía diferente a las demás. La última hoja de guarda está también numerada con número 303 .

- Hojas sin foliar: 92

- Tinta: color marrón

- Marca de agua: no la hemos podido encontrar en los libros de Vindel, Boffarull y Briquet sobre el tema ${ }^{1}$; lo único que podemos decir es que

1. BOFFARULL y SANS: Los animales en las marcas de papel, Villanueva y Geltrú, Oliva Impressor, 1910; BRIQUET, C.M.: Les filigranes, New York Hacker Art Books, 1966; VINDEL, F.: Escudos y Marcas de Impresores y Libreros, Barcelona, Orbis, 1942. 
las águilas son un motivo muy común, que proviene de Italia y que fue adoptado por la mayoría de los fabricantes de papel sobre todo desde Carlos I; asimismo hemos observado que los diseños recogidos por Boffarull son más simplistas y esquemáticos que el de S.C., que reproducimos a continuación:

La filigrana de la marca de agua no es perfectamente simétrica, como puede observarse en el dibujo, además no son todas idénticas, produciéndose leves variaciones en las alas de las águilas, en la cruz de la corona y en los círculos.

\section{Contenido del Manuscrito}

- Sonetos: Heróicos, 59

Amorosos, 19 numerados, 26 sin numerar. Laudatorios, 96

- Composiciones varias: 126

- Romances: 83

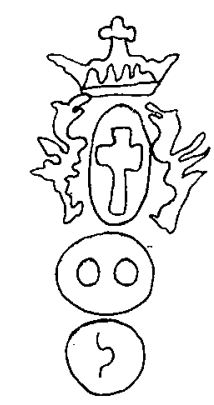

Van todos seguidos. El romance "La ciudad de Babilonia" está cortado; quedan 5 hojas sin foliar y en blanco entre el último verso que aparece del poema y el romance siguiente, lo que nos hace suponer que el copista reservó esas hojas para continuar en otra ocasión, cosa que no hizo.

\section{Abreviaturas utilizadas}

S.C.: Manuscrito B-3-9 de la Biblioteca del Real Seminario de San Carlos de Zaragoza, titulado Obras de Góngora.

Ch.: Ms. Chacón de las Obras de Luis de Góngora, de la Biblioteca Nacional de Madrid (Núm. Res./45).

Fuentes: Fuentes del Romancero General.

RG.: Romancero General.

Vic.: edición de Vicuña en 1627.

Del Par.: Delicias del Parnaso, Barcelona, 1630.

Durán: Colección del Romancero General, de su propiedad, Madrid, 1849-1851.

F.-D.: edición de Foulché-Delbosc.

H.U.: edición de Pedro Henríquez Ureña, basada en la de Foulché-Delbosc.

M.-G.: Millé y Giménez, Juan e Isabel, edición de 1934, reeditada en 1972.

Hozes: Todas obras... en varios poemas, recogidos por Don Gonzalo Hozes y Córdoba, Madrid, 1633.

P.A.E.: ed. de Adolfo de Castro en Poetas líricos de los siglos XVI y XVII, Madrid, B.A.E., XXXII, 1903, pp. 505-553.

A.S.: Ms. Aguilera Santiago, con el $n^{o} 141$ de la Biblioteca Menéndez Pelayo de Santander. 
F.: Obras de Don Luis de Góngora excepto el Polifemo, Soledades y Panegíricos, escritos de mano de Manuel Faria y Sousa; Ms. 2892 de la Biblioteca Nacional de Madrid.

Orden de los Romances de Góngora en el Ms. S.C.

1. ¿Quántos silbos, quántas vozes

2. Contando estaban sus raios

3. Las esmeraldas en yerba

4. Al tronco de un verde myrtho

5. Por las faldas de el Atlante

6. En los pinares de Xúcar

7. Los montes, que el pie se lavan

8. Apeóse el caballero

9. Al campo salió el estío

10. Las aguas de Carrión

11. Sin Leda y sin esperanza

12. En el baile de el exido

13. Amarrado al duro banco

14. La desgracia de el forçado

15. ¡O, quán bien que accusa Alcino

16. La cythara, que pendiente

17. Sobre unas altas rocas

18. En tanto que mis vacas

19. En el caudaloso río

20. Ciego que apuntas y atinas

21. Las flores de el romero

22. La más bella niña

23. Lloraba la niña

24. Ojos eran fugitivos

25. Donde esclarecidamente

26. Famosos son en las armas

27. En la fuerça de Almería

28. En un pastoral alvergue

29. Guarda corderos, zagala

30. No vengo a pedir silencio

31. Ilustre ciudad famosa

32. Aquel raio de la guerra

33. Según vuelan por el agua

34. No me bastaba el peligro

35. Esperando están la rosa

36. Aquí entre la verde juncía

37. Quatro o seys desnudos hombres

38. Ave de el plumaje negro

39. Frescos airecillos 
40. Entre los sueltos caballos

41. En lágrimas salgan mudos

42. Menguilla, la siempre bella

43. En dos luzientes estrellas

44. Saliéndome estotro día

45. Busqué para enamorarme

46. Trepan los gytanos

47. Tenemos un doctorando

48. Quando la rosada Aurora

49. Mançanares, Mançanares

50. Desde Sansueña a París

51. Tendiendo sus blancos paños

52. Dexad los libros ahora

53. Castillo de Sanct Cervantes

54. Murmuraban los rocines

55. Pensó rendir la moçuela

56. Despuntado he mil agujas

57. ¿Quién es aquel caballero?

58. En la pedregosa orilla

59. Ensílleme el asno rucio

60. Aunque entiendo poco griego

61. Arrojose el mançebito

62. De Thysbe y Pyramo quiero

63. La ciudad de Babylonia

64. Al pie de un álamo negro

65. Cloris, el más bello grano

66. A un tiempo dexaba el sol

67. Diez años vivió Belerma

68. Que necio que era yo antaño

69. Temo tanto los serenos

70. Ahora que estoy de espaçio

71. Triste pisa, y afligido

72. Hermana Marica

73. Hanme dicho hermanas

74. Tú noche, que alivias

75. Noble desengaño

76. Que se nos va la Pascua, moças

77. Moriste Nympha bella

78. Quantos silbas, quantas vozes

79. Nace el Niño, y velo a velo

80. ¿Quién oió?, ¿Quién oió?

81. ¡Quién pudiera dar un vuelo

82. De la semilla caída

83. En buen hora, o gran Phelipe

NOTA: Para el cotejo del Manuscrito del Seminario de San Carlos con el Manuscrito Chacón nos hemos servido de la edición de los Romances de 
Luis de Góngora preparada por Antonio Carreño (Madrid, Cátedra 1982), que sigue el ms. Chacón excepto en el poema "En el baile del ejido, / ¡nunca Menga fuera al baile!, cuya versión se basa en el Ms. Aguilera Santiago.

Como es sabido, Carreño anota variantes pertenecientes a otras fuentes; nosotros hemos consultado la edición de Pedro Henríquez Ureña (Góngora, Romances y Letrillas, Buenos Aires, Losada, 1939), que se basa en la edición de Raymond Foulché - Delbosc, realizada de acuerdo con el manuscrito Chacón y que consulta la versión de Juan e Isabel Millé Giménez (Madrid, hacia 1934) y también la edición de Madrid de 1654, que reproduce la igualmente madrileña de 1633.

Asimismo, hemos estudiado la versión de Millé Giménez en su edición de 1972 (Madrid, Aguilar).

Efectuado el cotejo de estas ediciones con la de Cátedra, creemos conveniente citar la relación de variantes que la edición de P. Henríquez Ureña y la de Millé Giménez presentan con respecto a la versión del Ms. Chacón que da A. Carreño y que no figuran anotadas estrictamente en su edición ${ }^{1}$ :

1. Ciego que apuntas y atinas / caduco dios, y rapaz v. 37 "sembré una estéril arena" M.G.=H.U.

4. Hermana Marica, / mañana, que es fiesta v. 9 "y a mí me pondrán” M.G. v. 69 "Jugaremos canas" M.G.

8. Diez años vivió Belerma / con el corazón difunto v. 33 "¿Qué culpa tuvistes vos" H.U. v. 105 "Escojamos como en peras" M.G. $=$ H.U.

9. En la pedregosa orilla / del turbio Guadalmellato v. 62 "de ver cuán a vivo tienes" M.G. v. 77 "si no quies verme difunto" M.G. $=$ H.U.

10. ¿Qué se nos va la Pascua, mozas, / que se nos va la Pascua! v. 24 "que traen la señal de la alba" M.G.

v. 34 "que fue en un tiempo rubia y zarca" H.U.

13. Aquí entre la verde juncía / quiero / (como el blanco cisne) v. 48 "a que siquieras me mires" H.U.

14. Aquel rayo de la guerra / Älférez Mayor del Reino v. 6 omitido en M.G. (ed. de 1972)

16. Entre los sueltos caballos / de los vencidos Cenetes v. 32 "satisface desta suerte" M.G.=H.U.

1. En esta relación seguimos el orden del Ms. Ch. y no el de S.C. Asimismo, sólo anotamos las variantes de los romances que aparecen también en el Ms. S.C., cuyo estudio es el objetivo de este trabajo. 
v. 51 "una dama de linaje" H.U.

v. 73 "Esta español, es la causa" M.G.=H.U.

v. 80 "que paren sus males quiere" M.G.=H.U.

v. 87 "que cupiera alma tan tierna" M.G.=H.U.

v. 91 "que me pedirán por robo" M.G.=H.U.

v. 92 "la que entendía que era suerte" H.U.

v. 100 "pido que de mí te acuerdes" M.G.=H.U.

v. 107 "que ganas más con librarme" M.G.=H.U.

19. Ahora, que estoy despacio,/ cantar quiero en mi bandurria No consta ni en M.G. ni en H.U.

22. Ilustre Ciudad famosa / infiel un tiempo, madre Dedicatoria en H.U.: A la ciudad de Granada" v. 75 "entran a adorar a quien" H.U.

v. 116 "beben, y la del Henares" M.G.

v. 124 "el gran Gonzalo Fernández" M.G.

v. 184 "y al Jaragüí, donde espiran" H.U.

24. Hanme dicho, hermanas / que teneis cosquillas

H.U. lo titula "Autorretrato"

v. 258 "el gusto en campillas" H.U.

26. Pensó rendir la mozuela / el Alférez de mentira v. 57 "Soy el de Santo Domingo" M.G.

v. 69 "donde padecía peligros" H.U.

27. Arrojose el mancebito / al charco de los atunes v. 25 "Mas al animoso joven" M.G.

v. 66 "la vez que se lo descubren" M.G.=H.U.

v. 72 "pastillas de piedra sufre" H.U.

28. Famosos son en las armas / los moros de Canastel v. 42 "(tan extrema en el correr" M.G.

29. Frescos airecillos / que a la Primavera

v. 15 "medias de esmeralda" M.G.=H.U.

v. 100 "y arrastrar cadenas" M.G.

32. Dejad los libros ahora, / señor licenciado Ortiz

v. 77 "preguntadlo a mi vestido" M.G.=H.U.

v. 105 "Dejela a su antiguo lustre" M.G.

v. 110 "mercadante crochapín" M.G.

33. ¿Qué necio que era yo antaño, / aunque hogaño soy un bobo v. 24 "cuando va de cuerdo a loco" H.U.

35. Tendiendo sus blancos paños / sobre el florido ribete v. 57 "Violante, que en un tiempo fuiste" H.U.

40. Moriste, Ninfa bella, / en edad floresciente v. 57 "(el Tiempo, tus memorias" H.U. 
41. Sin Leda y sin esperanza / rompe en mal seguro leño v. 31 "Lágrimas vierten tus ojos" H.U.

42. Despuntado he mil agujas / en vestir a Moriscote v. 20 "las espaldas al azote" M.G.=H.U.

v. 52 "Plegue a Dios que se derrote” M.G.

v. 65 "Dejadle vuelva a jugar" M.G.

v. 75 "se tocará lienzo crudo" M.G. =H.U.

43. Temo tanto los serenos, / serenísimo compadre v. 12 "no hay gracia ni habrá sepades" M.G. v. 29 "Mas al fin en estas cartas" H.U.

44. ¿Quién es aquel caballero / que a mi puerta dijo: Abrid? v. 45 "Gentil-hombre hice a muchos" H.U.

45. Las aguas de Carrión, / que a los muros de Palencia v. 13 "en el coro de Diana" M.G.

47. En tanto que mis vacas / sin oillos condenan v. 3 "en fruto los madroños" H.U.

48. En un pastoral albergue / que la guerra entre unos robles v. 49 "enfrénanle de la bella" M.G.=H.U.

v. 81 "Corona un lascivo enjambre" M.G. =H.U.

v. 98 "son tus roncos atambores" H.U.

v. 102 "vuela el caballo sin orden" M.G.

v. 104 "con jazmines si le coge" M.G.=H.U.

49. Según vuelen por el aire / tres galeotas de Argel v. 9 "Mortal caza viene dando" H.U.

52. En los pinares de Júcar / vi bailar unas serranas v. 32 "honestamente levanta" M.G.=H.U.

54. Cuando la rosada Aurora / o violada si es mejor v. 14 "mas él, de un buen pagador" M.G.

55. De Tisbe y Píramo quiero, / si quisiere mi guitarra v. 35 "dulce pero simple gente" M.G.=H.U. v. 63 "en favor lo que dicen" H.U.

60. En el baile del ejido, / („nunca Menga fuera al baile!) v. 16 "quien busca corales?" M.G.=H.U.

61. Esperando están la rosa / cuantas contiene un vergel v. 81 "en el murmurar continuo" H.U.

62. Apeóse el Caballero / (víspera era de San Juan) v. 44 "mi libertad te espera cada día" M.G. $=$ H.U.

63. Saliéndome estotro día, / candidísimo Lector v. 16 "las rejas de su prisión" M.G.=H.U.

v. 21 "Que el vaso de oro, en que os sirve" H.U. v. 53 "pegándole ella en madera" H.U. 
65. Tenemos un Doctorando, / discretos y generosos v. 25 "sino por oblicuidad" H.U.

v. 64 "lo tiene como un madroño" H.U.

v. 83 "que yo de explicarles puntos" H.U.

v. 104 "A la Q. de nuestro loco" H.U.

v. 137 "soñando al fin vuestro nombre" H.U.

67. No vengo a pedir silencio / que la Cómica Española v. 90 "lenguas sean de tus obras" H.U.

v. 93 "De esta santa iglesia hable" H.U.

v. 96 "de sus reliquias custodia" H.U.

68. ¡Cuántos silbos, cuántas voces / la nava oyó de Zuheros v. 56 "tanta precaución o miedo" H.U.

v. 65 "Los cuernos del Sol la dora" H.U.

69. De la semilla caída, / no entre espinas ni entre piedras

v. 12 "cuando no halda de jerga" H.U.

v. 47 "en nombre iguales, el fue" H.U.

v. 92 "y espumas de Tornes selían" H.U.

v. 101 "cuyos cilicios limando" H.U.

v. 119 "nos dejó Antonio. Produzga" H.U.

73. Al pie de un álamo negro, / y más que un negro bozal

v. 2 "y más que negro bozal" H.U.

v. 67 "primadas fueran de España" H.U.

v. 94 "donde tantos Pares hay" H.U.

v. 106 "llevando más de real" H.U.

77. Ojos eran fugitivos / de un pardo escollo dos fuentes v. 7 "entre concentos de llanto" H.U.

78. Manzanares, Manzanares, / vos, que en todo el acuatismo v. 31 "que lame en su piel diamantes" H.U.

79. Cuántos silbos, cuántas voces / tus campos, Bethlén, oyeron v. 18 "en vez de abarcas el viento" H.U.

81. Al tronco de un verde mirto, / enamorado Fileno v. 21 "suenen las trompetas, suenen" H.U.

v. 36 "que si el bien saben que espero" H.U.

82. En la fuerza de Almería / se disimulaba Hacén v. 12,13 ... "Celidaja" H.U. v. 49 "Pliega el dorado volumen" H.U.

83. Por las faldas del Atlante, / no como precipitado v. 17 "do el Amor fomenta el fuego" H.U.

84. Minguilla la siempre bella, / la que bailando en el corro v. 37 "Con más aliento aquel mayo" H.U.

87. Guarda corderos, zagala, / zagala, no guardes fe v. 11 "a pesar de su dureza" H.U. 
90. La cítara que pendiente / muchos días guardó un sauce v. 30 "a esta lisonja que hacen" H.U.

v. 45 "Quiérame la aurora" H.U.

91. Ave de plumaje negro, / si bien de tanto esplendor v. 1 "Ave del plumaje negro" H.U.

92. Nace el Niño, y velo a velo / deja en cabello a su Madre v. 15 "colcos de preciosa lana" H.U.

Para el cotejo de los romances que señala Carreño y Millé también como atribuíbles, "En buen hora, oh gran Phelipe" y "Tú, noche que alivias", hemos consultado la edición de la Biblioteca de Autores Españoles (B.A.E.). Sobre los poemas atribuíbles constatamos lo siguiente:

- En buen hora, oh gran Phelipe: aparece editado por F.-D. y recogido en la B.A.E.; también en Todas obras de Don Luis de Góngora, colección hecha por Don Gonzalo de Hozes y Córdoba, con el número 142.

- Tú, noche que alivias: aparece en A. de Castro (CIX), F.-D., Barc, 130 v, F, 177, Hozes, 130r, Del. Par. 107.

- Busqué para enamorarme: lo documenta Simón Díaz en POESIAS Salvé y Mallén, Pedro: Catálogo de la Biblioteca de Salvá; Tomo I, Valencia, 1872, pp. 238-240; también en Barc., 312, F., 149.

\section{Relación de variantes nuevas que presenta el Ms. S.C.}

1.- ¡Quántos silbos, quántas vozes / la Nava oió de Zuheros! (fol. 151 v)

En S.C. no aparece la dedicatoria presente en Ch.

v. 3: "sentidos bien de sus valles"

v. 27: "piñascos"

v. 44: "queditico"

v. 46: "obscuro"

v. 47: "saphyro"

v. 74: "paçer las que trençó flores"

2.- Contando estaban sus raios / aun las más breves estrellas (fol. $152 \mathrm{v}$ )

v. 32: "y quanto escucha desdén"

v. 81: "monstro"

S.C. (igual que Vic.) omite el verso $10 \mathrm{de}$ Ch.

3.- Las esmeraldas en yerba, / los alcáçares, de quien (fol. 154 r)

Dedicatoria: "A los Reyes de Hespaña, Felipe $4^{\circ}$ y Isabela de Borbón"

v. 6: "despreçió por lo clavel"

v. 19: "tronco en la legalidad"

v. 21: "iQuántos effectos les deben"

v. 22: "los ecos de Aranjuez" (igual que M.-G) 
v. 24: "suspiros fueron después"

v. 30: "si en sus márgenes se veen"

v. 34: "que los cysnes de la espuma"

4.- Al tronco de un verde myrtho / enamorado Phileno (fol. 155 r)

Dedicatoria: "A los mismos"

v. 8: "entre cuydados Rugeros"

v. 11: "horas en el mar prolixas"

vs. 15-16: "que cerrados ya los ojos/ aun más Cupido es que el ciego"

v. 24: "envestidles"

v. 28: "aunque las perdona el viento"

vs. 29-30: "que el fomentar su tardanza / disminuir es su vuelo"

v. 35: "Las horas vuelven atrás"

v. 36: "que si el bien saben que espero"

S.C. coloca los siguientes versos al final del último de Ch. (igual que B.A.E.):

I edra vividora,

dichosa vestia

luziente alcaría

de aquel sol que adora

garzón siempre bello,

que un cordero al cuello

su ganado es;

a esta iedra, pues

fía el sueño breve

quando perlas bebe

la causa en las flores,

quando ruiseñores

en el myrtho verde

recuerde, diçen, recuerde

quien amores tiene

que un sol con dos soles viene.

Dulce más que el arroiuelo

que las açucenas pisa,

llegó Belisa.

De raios se bordó el cielo,

y el zagal

aunque es Aguila Real,

su luz apenas sostiene

-que un sol con dos soles viene-

Gallardo más que la palma

que besa el aire sereno,

salió Phileno.

En sus ojos salió el alma

a recibilla,

y amorosa tortolilla 
hiço el caso más solene

-que un sol con dos soles viene-.

5.- Por las faldas de el Atlante, / no como precipitado (fol. 156 r)

v. 11: "comiença en crystal corriente"

v. 26: "mas nada lo tiene vano" (Ch. v. 14)

v. 51: "diçe bien" (Ch. v. 39)

v. 60: "a la melodía" (Ch. v. 48)

Todos los Mss. consultados por Carreño y también la edición de la B.A.E. omiten los siguientes versos que aparecen en S.C, entre los 12 y 25 (Ch. 12 y 13):

Plantas lo beben y yerbas, mas en differentes vasos, de pórfidos unas duras, de jaspes otras manchados.

Varias figuras informa y a no informar hoy él tanto

Alpheo no fuera río ni Arethusa fuera mármol.

Sus convecinos leones en bronçe huelga, que quanto lo bebieron ya sangrientos lo restituían dorados".

6.- En los pinares de Xúcar / vi bailar unas serranas (fol. 157 v)

v. 12: "por besar de ellas las plantas"

v. 26: "al saphyro, o la esmeralda"

v. 27: "El pie (quando lo permitte)" (igual en Vic. y en M.-G.)

v. 32: "honestamente levanta"

v. 38: "hiriendo lisas pizarras" (igual en Vic. y en Durán)

v. 40: "que las musas lo invidiaron" (igual que en Vic. y Durán)

S.C. y Ch. difieren en el orden de las dos últimas estrofas (vs. 49-56, 57-64)

7.- Los montes, que el pie se lavan / en los crystales de el Tajo (fo $1.158 \mathrm{v}$ )

v. 15: "lo dexó la blanca luna"

v. 16: "y lo halló el luziente Phebo"

v. 23: "a quán se deben sus raios" (igual en Durán)

v. 50: "-dice el pastor sin aliento-"

8.- Apeóse el caballero / (víspera era de Sant Juan) (fol. 159 v)

v. 7: "le sirve en la fuente de plata"

v. 25: "disafía"

v. 31: el texto dice por error "me libertad te espera cada día", en lugar del posesivo $m i$.

v. 35: "y embraçándolo pavés" 
v. 51: "embebida ya en el choro"

v. 59: "convirtió el color morado" (igual en Vic. y Durán)

v. 100: "la haçe deçir asî"

v. 106: "de las ancas de el rocín"

Hay un cambio de orden: los versos 51 y 52 aparecen trastocando su posición con los 55 y 56 con respecto al Ms. Ch.

9.- Al campo salió el estío / un seraphín labrador (fo l. $161 \mathrm{v}$ )

No presenta variantes.

10. Las aguas de Carrión / que a los muros de Palencia (fol. 162 r)

v. 4: "o espejo de sus almenas"

v. 13: "en el coro de Diana"

v. 15: el texto dice "horido" por "herido"

v. 25: "Más acrecientan sus ojos" (igual en Vic.)

11. Sin Leda y sin esperanza / rompe en mal seguro leño (fol. 163 r)

v. 23. "Porque el Amor lo assegura" (igual que B.A.E.)

v. 31: "Lágrymas verten sus ojos"

12: En el baile de el exido / nunca Mença fuera al baile (fol. $163 \mathrm{v}$ )

Utiliza Carreño en este poema la versión del manuscrito Aguilera Santiago, propuesta por Jammes como la más original; de igual manera, nosotros usamos como base de comparación para este poema el citado manuscrito (siguiendo la edición de Carreño) y no el Ch.

v. 4: "un dissanto por la tarde" (igual que Durán)

v. 12: "llora Menguilla crystales"

v. 16: quien busca corales?"

v. 40: "hazer las letras más grandes" (igual que Ch. y M.-G.) A.S. v. 24

v. 41: "quando albricias pidió a vozes" (igual que Ch. y M.-G.) A.S. v. 25

v. 44: "en sus labios sus corales" (igual que Ch. y M.-G) A.S. v. 28

v. 45: "los ojos fueron de antojos" A.S. v. 37

v. 47: "en la juncía los claveles" A.S. v. 39

v. 48: "en la arena los granates" A.S. v. 40

v. 51: "al son dixo de el psalterio" (igual que Ch. y M.-G) A.S. v. 43

v. 52: "que tañía Gil Perales" (igual que Ch. y M.-G) A.S. v. 44

S.C. repite el primer estribillo en las tres ocasiones.

S.C. (igual que Ch.) traspone la estrofa "En el exido los buscan" colocándola tras el verso 20, y siguiendo a continuación dos estrofas que no aparecen en A.S. pero sí en Ch. y en la edición de la B.A.E.:

Do en pago de su blancura menosprecian arrogantes las blancas espumas, que orlan el verde y florido margen.

Que la nieve es sombra obscura, 
y el marfil, negro azavache, con la garganta de Menga, columna de leche y sangre.

13.- Amarrado al duro banco / de una galera turquesca (fol. $164 \mathrm{v}$ )

v. 29: "Pero, pues no me responde"

14. La desgracia de el forçado / y de el corsario la industria (fol. $165 \mathrm{v}$ )

v. 29: "que quantos fueren mis años" (igual que Fuentes, V, VIII, RG.)

v. 38: "Y favorécelo el tiempo"

v. 40: "quanto de mi cautiverio"

15. ¡O, quán bien que accusa Alcino, / Orpheo de Guadiana (fol. 166 r)

13: "La vida es corta, la esperança larga"

16.- La cythara, que pendiente / muchos días guardó un sauze (fol. $166 \mathrm{v}$ )

v. 15: "porque inmudeçió los soplos"

v. 24: "aplausos hiço fragrantes"

v. 34: "invidia de los zagales"

v. 37: "manteniendo, pues, los ojos"

v. 38: "dulçen", error ortográfico por "dulçes"

v. 50: "El Alva me invía"

v. 64: "saludo otro albor"

17.- Sobre unas altas rocas / exemplo de firmeça (fol. 168 r)

v. 24. "al son de mil querellas"

v. 35: "de que tú no las pises" (igual que Vic., Hozes)

v. 41: "se aquesta se te escapa", error por "si aquesta"

v. 43-44: "dime, ¿qué te aprovechan / los filos de tus alas" (igual que Vic.

Hozes, Durán).

18.- En tanto que mis vaccas / sin oillos, condenan (fol. $168 \mathrm{v}$ )

v. 5: "quiere cantar llorando"

v. 10: "e intricadas trepas"

19.- En el caudaloso río / donde el muro de mi patria (fol. $169 \mathrm{v}$ )

v. 4: "y el antiguo pie se baña" (igual que Fuentes, RG., Vic.)

v. 10: "mirávalo su amor desde la orilla"

v. 16: "do su natural los llama"

v. 18: "los otros desde la barca"

v. 41: "volad al cielo, suspiros" (igual que RG., Vic. Durán) 
20.- Ciego que apuntas y atinas, / caduco dios, y rapaz (fol. $170 \mathrm{v}$ )

v. 7: "invidia"

v. 47: "miel dulce al amaro acíbar"

21.- Las flores de el romero / niña Isabel (fol. $171 \mathrm{v}$ )

v. 7: "dichoso, pues lo buscas"

v. 22: "te eclypsa tu placer"

vs. 27-28: "Enxuguen esperanzas / lo que lloras por él"

22.- La más bella niña / de nuestro lugar (fol. 172 r)

v. 4: "aier por casar" (igual que Vic.)

v. 48: "de mi soledad"

v. 58: "sobra la midad" ("midad" por "mitad", error ortográfico)

23.- Lloraba la niña / (y tenía razón) (fol. 173 r)

v. 6.: "que apenas creió"

24.- Ojos eran fugitivos / de un pardo escollo dos fuentes (fol. $174 \mathrm{r}$ )

v. 14: "a la montaña se atreven"

v. 19: " como un alamar de plata"

v. 32: "disvaneçido sin veerse"

v. 40: "en el ámbitu de el Betis"

v. 52: "quatro o seys flechas desmiente" (Ch. v. 48)

v. 54: "a los campos más reçientes" (Ch. v. 50)

v. 75: "tras un ciervo, que no huie" (Ch. v. 71)

v. 73: "con media luna de el sol" ( $\mathrm{CH}$. v. 69)

vs. 77-78: "Engañó a la caçadora, / conducido de esta suerte"

Los versos 45-48 se trasponen en Ch. en el orden 73-76.

25.- Donde esclarecidamente / guarnecen antiguas tores (fol. $175 \mathrm{r}$ )

S.C. omite la dedicatoria

v. 44: "Perlas da que lo coronen"

26.- Famosos son en las armas / los moros de Canastel (fol. $176 \mathrm{v}$ )

v. 8: "en Ceuta de el portugués"

v. 15: "le despedió Beleripha"

v. 29: "Mirábalo en los ramblares"

v. 37: "Mirábalo el más galán"

v. 84: "sin sabelle responder"

27.- En la fuerça de Almería / se dissimulaba Haçén (fol. 178 r)

v. 5: "Entre el cuchillo y la cuna" (igual que Durán y M.-G.)

v. 8: "que lo bastó defender" 
v. 9: "Negando, pues al rigor" (igual que M.-G.)

v. 12 (y 13 y 83): "Celidaxa"

v. 31: "fragrantes"

v. 48: "de un árbol, que ni Lucreçia"

v. 49: "Pliega el dorado volúmen"

v. 91: "desvaneçió, y en su forma"

Los versos 85-88 de Ch. faltan en S.C. y también en Durán.

28.- En un pastoral alvergue / que la guerra entre unos robres (fol. $179 \mathrm{v}$ )

v. 2: "que la guerra entra unos robres", debería decir "entre"

v. 12: "lo coronó de favores"

v. 15: "lo halló en el campo aquello"

v. 37: "Yerbas le applica a sus llagas"

v. 49: "Enfrénanle de la bella"

v. 66: "do a una labradora acoge"

v. 79: "invidia"

v. 81: "Corona un lascivo enxambre"

v. 94: "su vestido espira dores"

v. 129: "Choca, pues, tálamo y lecho", debería decir "choça"

29.- Guarda corderos, zagala / zagala no guardes fee (fol. 182 r)

v. 11: "a pesar de su dureça" (igual que Durán y M.-G.)

v. 12: "obedeçen al sinzel" (igual que F.-D.)

v. 21: "Tortolilla gruñidora"

v. 24: "las ojas de aquel ciprés"

v. 25: "La inconstancia le da plumas" (Ch. v. 33)

v. 46: "honrares al sol, y quien"

v. 58: "de tu hermosura cruel" (igual que Durán y M.-G.)

Ch. trastoca la estrofa $25-2,8$ ("la inconstancia, al fin, da plumas") y la coloca tras el v. 36.

30.- No vengo a pedir silencio, / que la cómica hespañola (fol. $183 \mathrm{v}$ )

Dedicatoria: "A Don Diego de Mardones, obispo de Córdoba, queriendo haçer una comedia en su presençia sus criados, echó uno esta loa"

v. 7: "monstro"

v. 17: "Y a mí en plectro agradeçido"

v. 32: "y breve rato, o perdona" (igual que Vic.)

v. 54: "el candelero a la entorcha"

v. 65: "invidia"

v. 66: "Phelipe a tus pies se prostra"

v. 90: "lenguas sean de tus obras"

v. 108: "a que yo os convido ahora" 
31.- Ilustre ciudad famosa / infiel un tiempo, madre (fol. 185 r)

Dedicatoria: "A la ciudad de Granada, y a sus grandeças, estando en ella Don Luis"

v. 13: "traxiste"

v. 16: "alcaides"

v. 21: "Y a veer de tu fuerte Alhambra" (igual que Fuentes, RG., Vic.)

v. 36: "Hybiernos"

v. 63: "echan a culpas de cera" (igual que Vic.)

v. 68: "y la natura de el arte" (igual que Vic.)

vs. 74-75: "por do los raios solares / entran a adorar a quien"

v. 116: "beben y la de el Henares"

v. 139: "Raynaldos"

v. 145: "y a veer tu Albaizín, castillo"

v. 160: "dos mis vistosos ultrajes"

v. 163: "Que dirán, se no se mueven" ("se no" por 'si no')

v. 164: "que es por faltarle el aire"

v. 166: "que al Darro çanefa haçen" ("çanefa" por 'cenefa')

v. 169: "Do el zephyro al blanco chopo" (igual que Fuentes y RG.)

v. 173: "donde hay de árboles tan greña"

v. 180: "porque los troncos se bañen"

vs. 185-186: "y al xaraguí, donde espiran / dulce olor los frescos valles"

v. 189: "cuyo solo viste Flora"

V. 200: "Y al rico Tajo deshaçes"

v. 203: "invidia"

v. 204: "eclypsi"

v. 210: "que dirán que entre sus perlas" (igual que Fuentes, RG., Vic.)

v. 212: "distila Amor sus paneles"

v. 218: "con un deseo notable" (Fuentes, RG., Vic.)

v. 225: "Y que se passen por veellas"

v. 236: "Daphes" por 'Daphnes'

32.- Aquel raio de lo guerra / Alférez mayor de el Reyno (fol. 189 v)

v. 23: "a que lo condena el rey"

v. 24: "o el amor que es el mar çierto"

v. 35: "lo manda destierrar luego"

v. 37: "para desculpar sus zelos"

v. 46: "tan gallardo iba il caballo" ("il" por 'el')

v. 66: "No lleva más que un alfange"

v. 76: "Y las damas, por do passa"

v. 91: "y en tu ausençia tien consuelo"

v. 93: "no te echerá de mi pecho" ("echerá" por "echará')

v. 102: "tornó el camino derecho"

33.- Según vuelan por el agua / tres galeotas de Argel (fol. 191 v)

v. 7: "timor"

v. 21: "Derrotolo un temporal" (Ch. v. 17) 
v. 33: "Ya sulcan el mar de Denia" ("sulcan" por 'surcan') (Ch. v. 29)

v. 43: "envuelven de negra humo" (Ch. v. 39)

v. 54: "húmida" (Ch. 50)

v. 56: "Seños de naufragios den" ("Seños" por 'señas') (Ch. v. 52)

Los versos 17-20 de S.C. no se incluyen en ninguno de los Mss. consultados por Carreño; y tampoco en la edición de la B.A.E.:

"El Vergantín pues, dichoso

que tal mereçió tener

si no fue concha de Venus

concha de estas perlas fue"

34.- No me bastaba el peligro / de una grave enfermedad (fol. $192 \mathrm{v}$ )

v. 6: "a deprender a labrar"

v. 8: "y un suave perdonar" (igual que Vic.)

vs. 14-16: "si vais para la ciudad / y acaso os mettiere en ella / Amor o necessidad"

v. 24: "tiene dudoso lo más" (igual que Vic.)

v. 31: "que pisado entre sus flores"

v. 36: "traidores señas de paz" ("traidores" igual que Vic)

35.- Esperando están la rosa / quantas contiene un vergel (fol. $193 \mathrm{v}$ )

No hay epígrafe (igual que Vic.)

v. 9: "Como reyma de las flores" (igual que Vic.)

v. 10: "guardia la ciñe fiel"

v. 17: "No le hiçieron reverençia" (igual que Vic.)

v. 19: "immobilidad"

v. 20: "desculpa"

v. 42: "un Hyacintho y al papel"

v. 55: "siempre calça bonzeguí"

v. 64: "invidian"

36.- Aquí entre la verde juncía / quiero, como el blanco cysne (fol. $195 \mathrm{v}$ )

v. 3: "que envuelta en dulce harmonía" (igual que M.-G.)

v. 11: "Y pendiente de sus ramas" (igual que Durán)

v. 24: "si eres diosa o eres tigre"

v. 32: "robre"

v. 52: "de que tus pies no lo pises"

v. 53: "por el rastro que dexabas"

v. 74: "humido"

37.- Quatro o seys desnudos hombres / de dos escollos o tres (fol. 197 r)

v. 12: "al piloto que lo vee"

v. 40: "de su hermosa cruel" (igual que F.-D.)

v. 56: "si al arco de Amor se cree" 
38.- Ave de el plumaje negro / si bien de tanto esplendor (fol. $198 \mathrm{r}$ )

v. 1: "Ave de el plumaje negro"

v. 12: "o prestar raios a un Dios"

v. 17: "Honesta, permittid ya"

v. 25: "quantas vezes remontada"

v. 33: "Visitolo, y si desierto"

v. 34: "lo halla mi devoçión"

v. 41: "O, reyna de cuanto vuela"

v. 42: "invidia"

v. 49: "Esto cantaba Feliso"

v. 50: "al dulçe luziente son"

39.- Frescos airecillos / que a la primavera (fol. 199 r)

v. 3: "destexeis guirnaldas" (igual que Vic. y Durán)

v. 24: "las verdes çanefas" (çanefas" por "çenefas')

v. 34: "invidia"

v. 62: "tremida en la sierra" ("tremida" por 'temida')

v. 86: "pero no tan cerca" (igual que Vic. y Durán)

40.- Entre los sueltos caballos / de los vencidos Cenetes [hoja $201 \mathrm{r}$ ]

v. 9: "Para que lo lleve a él"

v. 16: "que cuatro vientos le mueven"

v. 20: "y amaras lágrymas vierte"

v. 23: "que tan tiernamente llora"

v. 30: "sin escusarlo, obedece" (igual que Hozes)

v. 47: "después que murió mi padre" (igual que Hozes y Durán)

v. 48: "cossario"

S.C. tiene 72 versos igual que $\mathrm{Ch}$.

41.- En lágrimas salgan mudo / affectos, que hasta hoy [hoja $202 \mathrm{v}$ ]

v. 3: "aun en suspiros el alma"

v. 4: "el aire se las fio"

v. 10: "estrellas sin esplendor"

v. 19: "tan bella que él pide raios"

v. 34: "me hurta a la emulación"

Entre los versos 28 y 29 hay un blanco en el papel que coincide con el espacio de cuatro versos.

42.- Menguilla, la siempre bella / la que bailando en el corro [hoja 203 r]

v. 1: "Menguilla"

v. 9: "A esta pues deidad serrana"

vs. 10-11: "desde donde naçe arroio / hasta donde muere río" (igual que Prim. y Durán)

v. 17: "saphyros"

vs. 31-32: "tan pequeño de covarde/quan indistincto de ronco" 
v. 36: "Con más aliento que al Mayo"

v. 41: "en las ojas de la iedra"

v. 49: "Mientras distingue las fieras" Aparece en el margen derecho con otro tipo de escritura la palabra "extingue", a la vez que se subraya en el verso "distingue", es decir, en el manuscrito S.C. hay una corrección que marca otra variante.

v. 53: "Cambiarle hiçieron semblante" (igual que Prim. y Durán)

v. 54: "mas guardándole el decoro"

v. 64: "si no les pusiera cobro"

v. 95: "de que le negue un recato" (negue" por 'niegue')

v. 103: "Ya quería y de su espada" (igual que Durán)

v. 112: "que árbol es ya de Apolo" (igual que Prim. y Durán)

43.- En dos luzientes estrellas / y estrellas de raios negros [hoja $205 \mathrm{r}$ ]

Los versos 13 a 16 de S.C. no aparecen en Ch., Vic. y B.A.E. Se sitúan entre los versos 12 y 13 de los Mss. citados:

Y abrassada el alma mía

si contemplo en tal extremo,

fuego me dan por amor

y en satisfacción silencio.

44.- Saliéndome estotro día / candidíssimo lector [hoja 206 r]

v. 9: "Raboseando cuidados"

v. 16: "las rexas de su prission"

v. 20: "un ciutî"

v. 21: "que el vaso de oro, en que os sirve" (igual que Prim.)

v. 39: "siendo el Alva igualmente" (igual que Vic.)

v. 43: "si fue el respuenso de el muerto"

v. 52: "un marido, y sudeis vos" (igual que Vic. y Prim.)

45.- Busqué para enamorarme / las damas, que se usan hoy [hoja $207 \mathrm{r}$ ]

Este romance no aparece en el Ch. ni en ninguno de los otros Mns. consultados por Carreño para su edición; la composición es recogida como atribuible en Barc., fol. 312 v., Faria 149 r.: La versión de S.C. la transcribimos a continuación:

Busqué para enamorarme las damas, que se usan hoy, y de el más seguro estado no poco dudoso estoy.

Si soys donzellas, hay riesgo, si soys casadas mayor, si soys solteras, soys charcos de los sedientos de amor.

$\mathrm{Si}$ viudas, pareçeis urracas y si no os doy paga, qual diçe la urraca, 
deçís que soy ruiseñor.

Si soys damas de más de uno

no es perfecta mi affición

y si lo soys de mí sólo, pago al doblo y no lo soys.

Peor que peor

si soys de el prior.

Soys tan diestras en mentir que no hay engaño, o traiçión, que no lo desculpeis luego con apparente razón.

$Y$ si os cogen en las manos con el hurto qual ladrón, porque os den luego por libres buscais falsa información.

Si es moço, decís que es primo, si es ançiano, que es tutor, y si es fraile, por ventura que es Padre de confession.

Si es de el primo, a la prima no le faltará bordón, el anciano no es tan malo, mas de el frayle os guarde Dios.

Peor que peor si soys de el prior

No hay linze como vosotras con no ser hijas de el sol, mas soys de la luna hermanas mudables en condición.

Si ella se muda por quartos por quartos hay más de dos, que saben hazer mudanças más que tiene el esturdión.

Para mi bolsa en menguante tales lunas quiero yo quiero veerme en sus cuernos a quartos y no a doblón.

A la mejor de vosotras yo le echo mi bendición, que si soys buenas, soys malas y si soys malas peor.

Peor que peor si soys de el prior. 
46.- Trepan los gytanos / y bailan ellas [hoja 208 r]

v. 38: "que os asga la oreja" (igual que F.-D., Durán, Vic.)

v. 74: "desnudo lo deje"

v. 98: "legereças"

v. 107: "Fardos a Logroño"

v. 124: "hierramienta"

47.- tenemos un doctorando / discretos y generosos [hoja $210 \mathrm{r}$ ]

Dedicatoria: "Vexamen que dio en Granada a un Theólogo, dándole el grado de doctor. Era sobrino de el Administrador de el Hospital Real, donde están los locos; era pequeño de cuerpo, patón, bisojo y mal predicador. Diósele en día de Sanct Juan que caió aquel año en la infra octava de Corpus christi"

v. 8: "deçirla"

v. 17: "Hanos convidado a veerlo"

v. 19: "de los que lo están mirando"

v. 27: "tan serenos, que lo tienen"

v. 29: "doctoranducio"

v. 34: "venguençoso"

vs. $49,53,57,61$ : "conóçelo"

v. 64: "lo tiene como un madroño"

48.- Quando la rosada Aurora / o violada, si es mejor [hoja 213 r]

v. 3: "(escochan los epítetos)"

v. 5: "Las arboladas de Abril"

v. 21: "salió el sol, y halló el Machuelo"

v. 23: "que havían contado el dinero" (igual que Vic.)

v. 26: "si es macho cado varón" ("cado" por "cada')

v. 33: "Yo les doy, que passen esto"

v. 34: "que al macho desembolsó"

v. 35: "y en su lengua lo traduzgan"

v. 44: "Y assí lo fiantaron hoy"

v. 55: "oro engendre y cada piedra"

v. 93: "Fatigolo por el campo"

v. 94: "y después que lo cansó"

v. 105: "Sentió un no sé qué, y entiende" (igual que Vic.)

49.- Mançanares, Mançanares, / vos que en todo el aquatísimo [hoja 215 v]

v. 10: "Marqués de Poça el estío"

v. 13: "dezvanescais"

v. 17: "Enano soys de una puente"

v. 20: "le llegarais al tovillo"

v. 31: "que lame en su piel diamantes"

v. 32: "saphyros"

v. 63: "o sepulchro a los muertos" 
v. 64: "que no se comieren vivos"

v. 84: "y dien en fiado silbos"

v. 93: "que a fee que Reyes fallados"

50.- Desde Sansueña a París / dixo un medidor de tierra [hoja $217 \mathrm{v}$ ]

v. 2: "dixo un medidor de tierra"

v. 18: "Melisenda"

v. 19: "las que el griego llama nalgas"

v. 29: "con este escudero a pie"

v. 33: "Altravessaron a Hespaña"

v. 47: "para oir de el señor Pierros"

vs. 55-56: "valían veynte y cinco años / veynte y cinco mil de renta"

v. 80: "desempedrando la calle" (igual que Vic.)

v. 87: "la que Amor rindió a la puerta"

v. 88: "de el tiemplo de Sanct Dionys"

v. 93: "que la rasque la consçiencia"

v. 97: "y otro le quitó la nema"

v. 101: "mas que no saquen lançeta"

v. 108: "no sé quando le ablaban"

51.- Tendiendo sus blancos paños / sobre el florido ribete [hoja $220 \mathrm{r}$ ]

v. 9: "mosa de manto tendido"

v. 16: "dar tablas para un bofete"

v. 24: "lo dio a prueba de Mosquete"

v. 29: "Dexolo ya por un paje"

v. 40: "de un álamo su alcahuete" (igual que Vic.)

v. 44: "de el veynte y quatro Alderete"

v. 50: "de el lavadero, acomette" (igual que Vic.)

v. 72: "que lo lea, y que lo açete"

v. 76: "como torces tú un roquete"

v. 88: "el de los pardaguillete"

v. 98: "los niegras sienes le apriete"

v. 104: "la segundaba un puñete"

52.- Dexad los libros ahora / señor licenciado Ortiz [hoja $221 \mathrm{v}$ ]

v. 63: "los xaracandos respecto"

v. 64: "los modernos tahelí"

v. 69: "Sus pieças en el Hybierno"

v. 76: "quedé ermitaño Amadís"

v. 77: "Preguntadlo a mi vestido"

v. 110: "mercadante corchapín"

v. 112: "y ingenio en Mazalquivir" (igual que Fuentes) 
53.- Castillo de Sanct Cervantes / tú, que estás junto a Toledo [hoja 224 r]

v. 2: "tú, que estás junto a Toledo" (igual que Vic.)

v. 3: "fundote el rey don Alonso" (igual que Vic.)

v. 6: "mal fuerte, peor dispuesto"

v. 7: "pues que tienes mas padrastos"

v. 17: "Tiemplo fue (papeles hablen)"

v. 32: "que tienes prudentia al menos"

v. 48: "que tú tienes de Cervantes"

v. 52: "con dos dosenas de versos"

v. 55: "y por decirlo mejor"

v. 60: "y dulcísimo alimento" (igual que Vic.)

v. 69: "Dirásles que con tus años"

v. 82: "que recordará de el sueño" (igual que Vic.)

v. 94: "a sanct Angel y a Sanctelmo"

v. 98: "y no con tantos pertrechos" (igual que Vic.)

54.- Murmuraban los rocines / a la Puerta de Palaçio [hoja 226 r]

v. 5: "sino en su bestial idioma" (igual que Vic.)

vs. 23-24: "tan flacco en las carnes él / como yo enlas carnes flacco"

v. 28: "Ni la herradura con clavo" $\mathrm{Al}$ igual que Ch., S.C. presenta el dibujo

de un clavo en el verso, pero al margen escribe la palabra "clavo".

v. 45: "que piede al Consejo en paño" (igual que Vic.)

v. 51: "que es cólera de que escriben"

v. 53: "Sirvo, les dixo, a un pelón"

v. 56: "mas que dorme de prestado"

v. 77: "No sé como lo reçiben"

v. 85: "Deçiende luego tras ellos"

55.- Pensó rendir la moçuela / el alférez de mentira [hoja 229 r]

v. 43: "a no tenerlo ocupado"

v. 51: "alumbrando con su cara" (igual que M.-G.)

v. 62: "graçias a las Chavarrías"

c. 63: "inquieto fue desde niño"

v. 73: "quando en el sitio de Cypre"

v. 81: "y aun allí por gran ventura"

v. 107: "mas otra fuerça mayor"

v. 117: "Toda su oración prolixa"

56.- Despuntado he mil agujas / en vestir a Moriscote [hoja $231 \mathrm{r}$ ]

v. 8: "ni Albaialdos será bote" (igual que Vic.)

v. 16: "distilados de el cogote"

v. 20: "las espaldas al açote"

v. 43: "dicen que quiere probar"

v. 75: "se tocará lienço crudo"

v. 80: "se lo sirvan en gigote"

v. 81: "Dexadlo por vida mía" 
57.- ¿Quién es aquel caballero / que a mi puerta dixo: Abrid? [hoja 233 r]

v. 15: "un espino, dos romeros"

v. 24: "y en mi bolsa ni un ciutî"

v. 34: "me libraron un quartín" (igual que Vic.)

58.- En la pedregosa orilla / de el turbio Guadalmellato [hoja 234 r]

v. 11: "no más que por estar roto"

v. 14: "de el Amor, que lo ha matado"

vs. 23-24: el uno orillas de Esgueva / y el otro orillas de Darro"

v. 41: "Con lágrimas lo humedece"

v. 46: "habiéndolo antes besado"

v. 47: "lo envuelve y saca de el seno"

v. 77: "si no quies vierme diffuncto"

59.- Ensílleme el asno rucio / de el Alcalde Antón Llorente [hoja 235 v]

v. 22: "que en ciegos nudos los tienen" (igual que RG. y Durán)

v. 27: "ramos de nogal y espinas" (igual que RG., Vic., Durán, F.-D)

v. 36: "sin que los proveian los Reyes"

v. 43: "invidia"

60.- Aunque entiendo poco griego / en mis greguescos he hallado [hoja $237 \mathrm{v}$ ]

v. 28: "Hybiernos"

v. 31: "vara y no de vagabundos"

v. 80: "la muger de un Veynte y quatro"

v. 96: "hiçola ruedar qual pavo"

v. 99: "lo asseguró y le dio un bello"

v. 110: "y descuprió un sepan quantos"

v. 121: "los ojacos negros diçen" ("ojacos" por 'ojaços')

v. 128: "los pedió a Hespaña prestados"

v. 134: "mescló el Cielo un encarnado"

v. 136: "se lo hurtó al Alva aquel año

v. 140: "invidia"

v. 142 "y de el pecho el alabastro"

v. 170: "y tienénlo tan picado"

61.- Arrojose el mançebito / al charco de los atunes [hoja $241 \mathrm{v}$ ]

v. 10: "passaba con pesadumbre" (igual que Fuentes, RG., Vic.7

v. 22: "que ya suffrió como un iunque"

v. 23: "el exército de Xerxes"

v. 50: "el farol, que lo conduçe"

v. 54: "ya más vezes se zabulle"

v. 60: "a la orilla los acuden"

v. 65: "la vez que se lo descubren"

v. 71: "y el Alma donde se queman"

v. 72: "pastillas de piedra çufre"

v. 92: "y yo estrellada fin tuve" 
62.- De Thysbe y Pyramo quiero / si quisiera mi guitarra [hoja 243 r]

v. 35: "dulce pero simple gente"

v. 43: "niño de sus quatro ojos"

63.- La ciudad de Babylonia / famosa no por sus muros [hoja $244 \mathrm{r}$ ]

v. 3: "fuissen de tierra cocidos"

v. 61: "un rubí conciede o niega"

vs. 63-64: "entre doçe perlas nectas / veynte aljófares menudos" (igual que M.-G., F.-D., Vic.)

vs. 72-508: faltan en S.C. Hay cinco hojas en blanco entre el último verso de esta composición y el romance siguiente, lo que nos hace suponer que el copista reservó esas hojas para continuar en otra ocasión, lo que no hizo.

64.- Al pie de un álamo negro / y más que negro boçal [hoja $245 \mathrm{v}$ ]

v. 2: "y más que negro boçal"

v. 4: "sino gemir y callar"

v. 8: "dalle sus pies a beçar" ("beçar por 'besar')

v. 31: "lo va entrando en un confuso"

v. 41: "Congóxalo esto de suerte"

v. 67: "Primadas fueran de Hespaña" (igual que Vic.)

v. 92: "en Francia pude gastar"

v. 94: "donde tantos Pares hay"

v. 103-104: "que al que buscais con un peto/ lo hallais con un espaldar" (igual que Vic.)

v. 106: "llevando más de Real"

65.- Cloris, el más bello grano / sino el más dulce rubí [hoja 247 r]

v. 9: "fragrancia"

v. 20: "un ruiseñor carmesí"

v. 27: "juzgándolo quinta hoja"

v. 40: "y el cegueçuelo ruin"

66.- A un tiempo dexaba el sol / los colchones de las ondas [hoja $248 \mathrm{v}$ ]

v. 16: "las pagan a qué quien boca"

v. 33: "quando criminales y bella"

v. 54: "passos gasta y viento compra"

v. 61: "y lo halló entre unos carrizos"

v. 71: "Y en el envez de la Nympha"

v. 118: "y por lo que es verguençosa"

67.- Diez años vivió Belerma / con el coraçón diffuncto [hoja 251 r]

v. 30: "también lo quisistes mucho"

v. 36: "que lo entierren como a bruto"

v. 39: "que allí le hiçieron lugar" 
v. 46: "las tocas de angeo crudo" (igual que RG., Vic., Durán)

v. 58: "Sanctinuflo"

v. 69: "y no por eso ultrajé"

v. 75: "llévese el mar lo llorado"

v. 91: "ceja en arco, manos blancas" (igual que Vic.)

v. 96: "baterías y infortunios"

v. 105: "Escojamos como en peras"

68.- Que necio que era yo antaño / aunque ogaño soy un bobo [hoja $253 \mathrm{v}$ ]

v. 15: "captivos"

v. 18: "hizo un año por Agosto"

v. 27: "en tan verguençosas llamas"

v. 32: "aiudaré con un soplo"

v. 42: "ni pesadumbres mi ocio"

v. 65: "Y a la que vio Pisuegra"

v. 71: "y a dar verde al pensamiento"

v. 92: "monstro"

v. 98: "affirmado el tiempo coxo" (igual que Vic., M.-G.)

v. 99: "sobre un sceptro Imperial"

69.- Temo tanto los serenos, / sereníssimo compadre [hoja $255 \mathrm{v}$ ]

S.C. no tiene dedicatoria.

v. 8: "no se entendíen con el aire"

v. 12: "no hay gracia, ni havrá sepades"

v. 22: "que padecieron tres naipes" (igual que Vic.)

v. 55: "y que el balcón de la alcoba"

70.- Ahora que estoy de espaçio / cantar quiero en mi bandurria [hoja $256 \mathrm{v}$ ]

v. 29: "en la Botiga otras vezes" ("Botiga" por 'Botica')

v. 30: "me daba muy buenas zurras"

v. 36: "que el mar de Bretaña sulcan" ("sulcan" por 'sulcan')

v. 58: "que en Pueblo tenía muchas"

v. 72: "como tisseras de murtas"

v. 106: "y que para el mal mandrugas" ("mandrugas" por 'madrugas')

v. 110: "no mostres en él tu furia" ("mostres" por 'muestres')

v. 119: "de gallina son tus alas"

71.- Triste pisa, y afligido / las arenas de Pisuerga [hoja $258 \mathrm{v}$ ]

v. 4: "disdichado"

v. 5: "Moro Alcaide y no Bellido"

v. 16: "cuyas plumas son tisseras"

v. 18: "cuyas ondas se lo llevan"

v. 19: "y envueltas entre las ondas"

v. 24: "acudiera en diez hanegas"

v. 41: "Ojos garzos, cejas rubias" 
v. 64: "más de novienta y seys leguas"

v. 66: "de una mugeril verguença"

72.- Hermana Marica / mañana que es fiesta [hoja 260 r]

v. 6: "la saia de seda"

v. 33: "con las dos hermonas" ("hermonas" por 'hermanas')

v. 35: "las dos primillas"

v. 39: "podrás tanto della"

S.C. y Ch. presentan trastocados los versos 16 y 24

73.- Hanme dicho hermanas / que teneis cosquillas [hoja $261 \mathrm{v}$ ]

v. 10: "filocomosía"

v. 21: "que como a las diez"

v. 64: "en rucia o tordilla" (igual que Vic.)

v. 91: "pues tienes (demás"

vs. 134-135: "de el Ave María / al quarto de el Alva"

v. 155: "o dosel lo cubra"

v. 156: "o adórnelo myrtho"

v. 164: "en la que le envía"

v. 166: "un gran canonista" (igual que Fuentes, RG., Vic., Durán)

v. 187: "que quien lo oie, diçe"

v. 190: "es tal, que dirían" (igual que Vic., RG., Fuentes)

74.- Tú noche, que alivias / los cansados miembros [hoja $266 \mathrm{r}$ ]

Este romance no aparece en el ms. Ch. Hacemos el cotejo con la versión de la edición de la B.A.E., en la que se dice que Rivas no lo cree de Góngora; asimismo, Carreño lo recoge como atribuible y da noticia de su presencia en A. de Castro (CII) y F.-D.

v. 4: "convidan con sueño"

v. 7: "de amorosos lazos"

v. 23: "tus trompetas roncas" (B.A.E. da noticia de esta variante sin concretar origen)

v. 27: "sin darme cuidado"

v. 33: "Ahora el cuidado"

v. 34: "usurpó tus fueros"

v. 35: "y entre tus tinieblas"

v. 45: "responsos a mi alma"

v. 51: "a mis esperanças"

v. 62: "contar mis sucessos"

v. 70: "Hybierno"

v. 86: "garzos, ni serenos"

v. 96: "temerosos zelos"

v. 133: "Ferviente en las causas"

v. 144: "y tygre en segreto"

v. 152: "pobre de contentos" 
v. 158: "que aunque no me duermo"

v. 160: "de esperanças secco"

75.- Noble desengaño / graçias doi al cielo [hoja 269 r]

v. 10: "el iugo de acero"

v. 45: "Porque el que más trata"

v. 50: "desde tu aposento" (igual que Vic., Durán)

v. 84: "Beltenebro"

v. 99: "Socorre, señora"

v. 108: "Hybierno"

Los versos 66 a 72 están trastocados con los 58 a 64 con respecto a Ch.

76.- Que se nos va la Pascua, moças / que se nos va la Pascua [hoja 271 r]

v. 45: "se lo dexó el otro día"

v. 50: "aunque ahora no soys nada" (igual que Vic., Hozes)

v. 56: "convierta en luziente nacar"

77.- Moriste Nympha bella / en edad floreciente [hoja $272 \mathrm{v}$ ]

Dedicatoria: "A la muerte de D. Luisa de Cardona, monja en Sancta Fee de Toledo"

v. 2: "en edad floreciente"

v. 45: "Turno gemido apenas"

v. 48: "no que lo llame ausente" (igual que Vic.)

v. 56: "su prescripción no entente"

v. 74: "en la inscripción siguiente"

78.- Quantos silbos, quantas vozes / tus campos, Belén, oieron [hoja 274 v]

v. 2: "tus campos, Belén, oieron"

v. 11: "los raios son de la luna"

79.- Nace el Niños, y velo a velo / dexa en cabello a su madre [hoja 275 r]

v. 8: "que aunque calientan, son aire"

v. 15: "colcos en preciosa lana"

v. 26: "fragrantes"

80.- “QQuién oió?, ¿Quién oió? / ¿quién ha visto lo que yo? [hoja 276 r]

v. 30: "Levánteme al harmonía"

v. 31: "y caiendo al resplandor".

v. 36: "que ni era ave, ni hombre"

v. 45: "al blanco zephyro hiçe"

v. 49: "llegué donde el ielo ví"

v. 51: "peinarle rayos al Sol"

vs. 58-59: "que me ha valido más luz / que la cáthedra mejor"

vs. 60-65: "Oí bailar un cordero / cordero que fue león / león, que si niño naçe / Amor divino / es niño, mas siempre Dios / divino Amor" 
81.- ¡Quién pudiera dar un vuelo / por todo lo que el sol mira [hoja 277 r] No presenta variantes.

82.- De la semilla caída / no entre espinas, ni entre piedras [hoja $277 \mathrm{v}$ ]

Dedicatoria: "A la Beatificación de Santa Teresa de Jesús"

v. 11: "sino castal de buriel"

v. 12: "quando no halda de xerga"

v. 24: "no se descalçó para veerla"

v. 33: "Baca pues, y en pocos años"

v. 76: "urna de su alma estrecha"

v. 83: "floresciente"

v. 108: "está distilando hoy muerta"

v. 126: "Trassiera"

83.- en buen hora, o gran Phelipe / volvais vuestra luz adonde [hoja $280 \mathrm{r}$ ]

No aparece en la edición de Carreño, quien lo cita como atribuible y da noticia de él en F.-D. De nuevo cotejamos las variantes con la versión de la edición de la B.A.E., donde también se dice que "Rivas Tafur no cree que sea de Góngora".

v. 1: "Phelipe" (igual en todos los demás casos)

vs. 9-12: "Ya el lusitano vio impuestos / gran Neptuno, Forte Jove,/ con el tridente y el sceptro / freno al mar, lei a los orbes"

v. 21: "de nuestros votos llamado"

v. 33: "No en dos veneras admiran"

v. 39: "vive calçando Hymeneo"

v. 58: "sceptro"

v. 60: "si no de diademas divinos"

v. 62: "haçe que a los dos el suelo"

v. 68: "los cultos en que la espera"

v. 80: "Decidles"

v. 84: "que de el segundo Phelipe"

v. 88: "que en las aras de una Virgen"

v. 92: "A las luzes consagrados"

v. 101: "que por ardentes regiones"

v. 103: "otro a las aguas dio nombre"

v. 111: "bañé de imaginaciones"

v. 113: "que inmortalidad compone"

v. 120: "la fami ví, que al siglo indeficiente"

v. 124: "Argos attentos discurrían mis ojos"

v. 134: "Aquel famoso rey, cuyas memorias"

v. 136: "faltaron palmas para tantas glorias"

v. 140: "Pues aun no bien a estas montañas frías"

v. 143: "en muchos siglos de una breve aurora"

v. 160: "mármoles consagró gloriosamente"

v. 163: "Hueste alada son cercos de su luna" 
v. 164: "occupaba después grande distançia"

v. 168: "mas al que no cedió mayor distançia"

v. 175: "Pompa todos mayor de la esculptura"

v. 189: "siglos, después aquel gran rey contemplo"

v. 190: "viven los fuegos de este sagro templo"

v. 204: "Joseph"

v. 218: "que eternizador en sus lumbres bellas"

v. 226: "Muerto murió que eterno ser recibe" (B.A.E. anota esta variante, pero no dice origen)

v. 231: "quantas ondas corrió crystal dorado"

v. 236: "Augusto en forma, en fee majestuoso"

v. 245: "Aras alçó, tan altos sus empleos"

v. 248: "No leo los nombres, informado el arte"

v. 264: "No leo los nombres, informado el arte"

v. 266: "o fama, si a tus glorias excedidas"

v. 267: "No son más que por ellas conocidas"

v. 269: "si arbolando la cruz nuevos pendones"

v. 280: "De el uno al levantar la altiva mano"

v. 283: "terras y mares les venían estrechos"

v. 294: "su rostro, que dos orbes ilustrando"

v. 300: "Salve te dixe o tú, que a dos Apolos"

v. 306: "que Pen podrás con passos tan seguros"

v. 317: "primera cuna quando a vos sea nido"

v. 324: "De aquestas digo luzes cinco hermosas"

v. 332: "Tú, que haciendo estos orbes firmamentos"

v. 335: "bañados de tu sol resplandeciente"

v. 336: "Oi, que estos montes ilustraste atentos"

v. 338: "vuelves feliz, entra estos patrios lares"

v. 348: "Arden las teas nupciales obedientes"

v. 349: "lilios la edad el thalamo perdone" (igual otros, según B.A.E.)

v. 353: "Por mas que el tiempo en mármoles blasone"

v. 364: "Dixe: quando de el tiempo crystalino"

v. 367: "Harmoniosos fastos dio a los cielos"

v. 374: "que me llevas los sentidos"

v. 385: "buscas eternas memorias"

v. 390: "cuyos despojos al templo"

v. 404: "Publicas affectos puros"

v. 405: "affectas luzientes pompas"

v. 407: "en desatados aromas"

v. 411: "erigió en sus aras proprias"

v. 412: "El sólo en ver sus altares"

v. 434: "Traiendo al sol de María" (B.A.E. 431)

v. 440: "la beldad de nuestra Infante" (B.A.E. 437)

v. 445: "porque a sus nombres respondan" (B.A.E. 442)

v. 450: "famosas en vuestras entradas" (B.A.E. 447)

v. 455: "aplausos humildes prostras" (B.A.E. 452) 
Dedicatoria: "Viniendo de Portugal el Rey don Phelipe Terçero por el mes de Octubre, anno de 1619, llegó a nuestra señora de Guadalupe, y a la entrada de la Iglesia havía un arco triunfal bien adornado, y en lo alto una nube, la qual fue baxando, quando su magestad llegó y, abriéndose se descubrieron dos figuras, que eran la Religión y la Justicia, y dixeron una a otra los versos siguientes, que son de Don Luis de Góngora".

Los versos 430 a 433 faltan en la B.A.E.:

"Y en las mismas ondas muere

ni la abrevia ni la toca

oy, que a este sagrado alcaçar

volveis las plantas devotas". 